Review Article

\title{
GLP-1 Receptor Agonist Effects on Lipid and Liver Profiles in Patients with Nonalcoholic Fatty Liver Disease: Systematic Review and Meta-Analysis
}

\author{
Shahla Rezaei, ${ }^{1,2,3}$ Reza Tabrizi, ${ }^{4}$ Peyman Nowrouzi-Sohrabi, ${ }^{1,5}$ Mohammad Jalali, ${ }^{1,3}$ \\ Stephen L. Atkin, ${ }^{6}$ Khalid Al-Rasadi, ${ }^{7}$ Tannaz Jamialahmadi, ${ }^{8}$ \\ and Amirhossein Sahebkar iD $9,10,11$ \\ ${ }^{1}$ Student Research Committee, Shiraz University of Medical Sciences, Shiraz, Iran \\ ${ }^{2}$ Department of Clinical Nutrition, School of Health \& Nutrition, Shiraz University of Medical Sciences, Shiraz, Iran \\ ${ }^{3}$ Nutrition Research Center, School of Nutrition and Food Sciences, Shiraz University of Medical Sciences, Shiraz, Iran \\ ${ }^{4}$ Noncommunicable Diseases Research Center, Fasa University of Medical Sciences, Fasa, Iran \\ ${ }^{5}$ Department of Biochemistry, School of Medicine, Shiraz University of Medical Sciences, Shiraz, Iran \\ ${ }^{6}$ Weill Cornell Medicine Qatar, Doha, Qatar \\ ${ }^{7}$ Medical Research Centre, Sultan Qaboos University, Muscat, Oman \\ ${ }^{8}$ Department of Nutrition, Faculty of Medicine, Mashhad University of Medical Sciences, Mashhad, Iran \\ ${ }^{9}$ Applied Biomedical Research Center, Mashhad University of Medical Sciences, Mashhad, Iran \\ ${ }^{10}$ Biotechnology Research Center, Pharmaceutical Technology Institute, Mashhad University of Medical Sciences, Mashhad, Iran \\ ${ }^{11}$ School of Pharmacy, Mashhad University of Medical Sciences, Mashhad, Iran \\ Correspondence should be addressed to Amirhossein Sahebkar; amir_saheb2000@yahoo.com
}

Received 5 August 2021; Revised 4 October 2021; Accepted 25 October 2021; Published 12 November 2021

Academic Editor: Manoj K. Sharma

Copyright ( 2021 Shahla Rezaei et al. This is an open access article distributed under the Creative Commons Attribution License, which permits unrestricted use, distribution, and reproduction in any medium, provided the original work is properly cited.

Aims. This meta-analysis of randomized placebo-controlled clinical trials assessed the effect of glucose-like peptide-1-receptor agonists (GLP-1RA) on the lipid profile and liver enzymes in patients with nonalcoholic fatty liver disease (NAFLD). Materials and Methods. Randomized placebo-controlled trials investigating GLP-1RA on the lipid profile and liver enzymes in patients with NAFLD were searched in PubMed-Medline, Scopus, Web of Science, and Google Scholar databases (from inception to January 2020). A random-effects model and a generic inverse variance method were used for quantitative data synthesis. Sensitivity analysis was conducted. Weighted random-effects meta-regression was performed on potential confounders on lipid profile and liver enzyme concentrations. Results. 12 studies were identified (12 GLP-1RA arms; 677 subjects) that showed treatment with GLP1RA reduced alanine transaminase (ALT) concentrations $(\mathrm{WMD}=-10.14,95 \% \mathrm{CI}=[-15.84,-0.44], P<0.001)$, gamma-glutamyl transferase $(\mathrm{GGT})(\mathrm{WMD}=-11.53,95 \% \mathrm{CI}=[-15.21,-7.85], P<0.001)$, and alaline phosphatase $(\mathrm{ALP})(\mathrm{WMD}=-8.29,95 \% \mathrm{CI}=$ $[-11.34,-5.24], P<0.001)$. Aspartate aminotransferase (AST) $(\mathrm{WMD}=-2.95,95 \% \mathrm{CI}=[-7.26,1.37], P=0.18)$ was unchanged. GLP-1 therapy did not alter triglycerides $(\mathrm{TC})(\mathrm{WMD}=-7.07,95 \% \mathrm{CI}=[-17.51,3.37], P=0.18)$, total cholesterol (TC) $(\mathrm{WMD}=-1.17(-5.25,2.91), P=0.57)$, high-density lipoprotein $(\mathrm{HDL}-\mathrm{C})(\mathrm{WMD}=0.97,95 \% \mathrm{CI}=[-1.63,3.58], P=0.46)$, or low-density lipoprotein $(\mathrm{LDL}-\mathrm{C})(\mathrm{WMD}=-1.67,95 \% \mathrm{CI}=[-10.08,6.74], P=0.69)$ in comparison with controls. Conclusion. The results of this meta-analysis suggest that GLP-1RA treatment significantly reduces liver enzymes in patients with NAFLD, but the lipid profile is unaffected. 


\section{Introduction}

Nonalcoholic fatty liver disease (NAFLD) is an increasing global public health problem with a worldwide prevalence of NAFLD estimated at approximately 25\% [1] and a common cause of chronic liver disease [2], and it is predicted to develop in more than $30 \%$ of the US adult population [3]. NAFLD is diagnosed when there is hepatic steatosis in the absence of other causes of hepatic fat [4]. In NAFLD, there is an accumulation of fat in the liver through increased free fatty acid delivery to the liver, increasing triglyceride synthesis, decreasing triglyceride export, and reducing betaoxidation [5]. Coexisting insulin resistance (IR) in NAFLD enhances lipolysis from the adipose tissue [5]. Currently, there are no approved drug treatments for NAFLD and NASH [6].

Glucagon-like peptide-1 receptor agonists (GLP-1RA) are a newly introduced class of antidiabetic drugs that improve glycemic control via several molecular pathways $[7,8]$. These pharmacologic agents reduce blood glucose via glucose-dependent insulin secretion and by glucagon suppression [8]. In addition, GLP-1RAs have other beneficial effects [9-16] and decrease energy intake and body weight by prolonging gastric emptying and inducing satiety [17]. There is an association between NAFLD and metabolic syndrome that causes DM, dyslipidemia, and obesity suggesting that breaking this cycle by GLP-1 agonists may have therapeutic potential [18], particularly as they may have anti-inflammation activity [19]. The administration of the GLP-1RA liraglutide was suggested to directly reduce liver fibrosis and steatosis in an in vivo study [17] and reduces markers of fibrosis in man [20]. Therefore, GLP-1 receptor analogue therapy may have the potential for the treatment of NAFLD and NASH patients; however, it is unclear from the studies that have been done whether GLP-1 agonists improve the hepatic enzyme and lipid profiles in subjects with NAFLD; therefore, this systematic review and meta-analysis were undertaken.

\section{Methods}

2.1. Search Strategy. This meta-analysis was conducted according to PRISMA instruction of systematic reviews and meta-analysis [21]. The scientific web-portals such as
PubMed, Scopus, Cochrane, Web of Science, Embase, and Scholar were carefully surveyed to extract all relevant literature on the effects of GLP-1 receptor agonists on lipid profile and liver enzymes in patients with nonalcoholic fatty liver disease published until January 2020. The key terms that were applied to finalize the first step of the search strategy to gather target data are shown in Appendix. Additionally, manual searches were performed to find articles that were not indexed in target databases. Only human-based studies were selected from the search strategy, and language restriction was not considered. Two authors (Sh.R. and P.N.) independently surveyed the title and abstracts of the classified papers, extracted relevant data, and applied quality assessments of eligible studies. A third author (R.T.) checked the data and resolved all disagreements.

2.2. Study Selection. The following strategy was utilized to select target papers: randomized clinical trials (parallel or cross-over) that investigated the effect of GLP-1 receptor agonists on the lipid profile and liver enzymes in patients with nonalcoholic fatty liver disease, individuals treated with GLP-1 receptor agonists that were compared with placebocontrol or other pharmaceutical agents, at least 12 weeks' administration of GLP-1 receptor agonists, papers that contained data for standard deviation (SD), standard error (SE), and confidence interval (CI) parameters in the beginning and the end of each study for both the intervention and control groups.

2.3. Data Extraction. Relevant RCT data were extracted by rechecking the name of first author, country, the number of individuals in the intervention and control groups, the type and doses of GLP-1 receptor agonists, duration of the study, type of the study, and related data for analysis (Table 1). For each study, the values of the mean and SD for lipid profile and liver enzymes were recorded at the beginning and the end of each study using the calculation of the difference between the values before and after the intervention. The following formula was used to calculate the mean difference of SDs:

$$
\mathrm{SD}=\text { square } \operatorname{root}\left[(\mathrm{SD} \text { baseline })^{2}+(\mathrm{SD} \text { end of study })^{2}-(2 r \times \mathrm{SD} \text { baseline } \times \text { SD end of study })\right]
$$

A correlation coefficient of 0.5 was used for $r$, estimated between 0 and 1 values [22]. The formula SD = $\mathrm{SE} \times \sqrt{n}$ ( $n=$ the number of individuals in each group) was used to measure SD in each article that reported SE instead of SD.

2.4. Quality Assessment. The quality assessment of the included papers in this meta-analysis was conducted based on Cochrane criteria [23]. Accordingly, any source of bias, including selection bias, performance bias, detection bias, attrition bias, and reporting bias, was judged for all included studies (Figure 1).

2.5. Statistical Analysis. A random-effects model was performed using Stata v.13 (StataCorp. 2021, Stata Statistical Software: release 17; College Station, TX: StataCorp. LLC) to obtain weighted mean difference (WMD) and corresponding $95 \%$ CIs. Interstudy heterogeneity was 


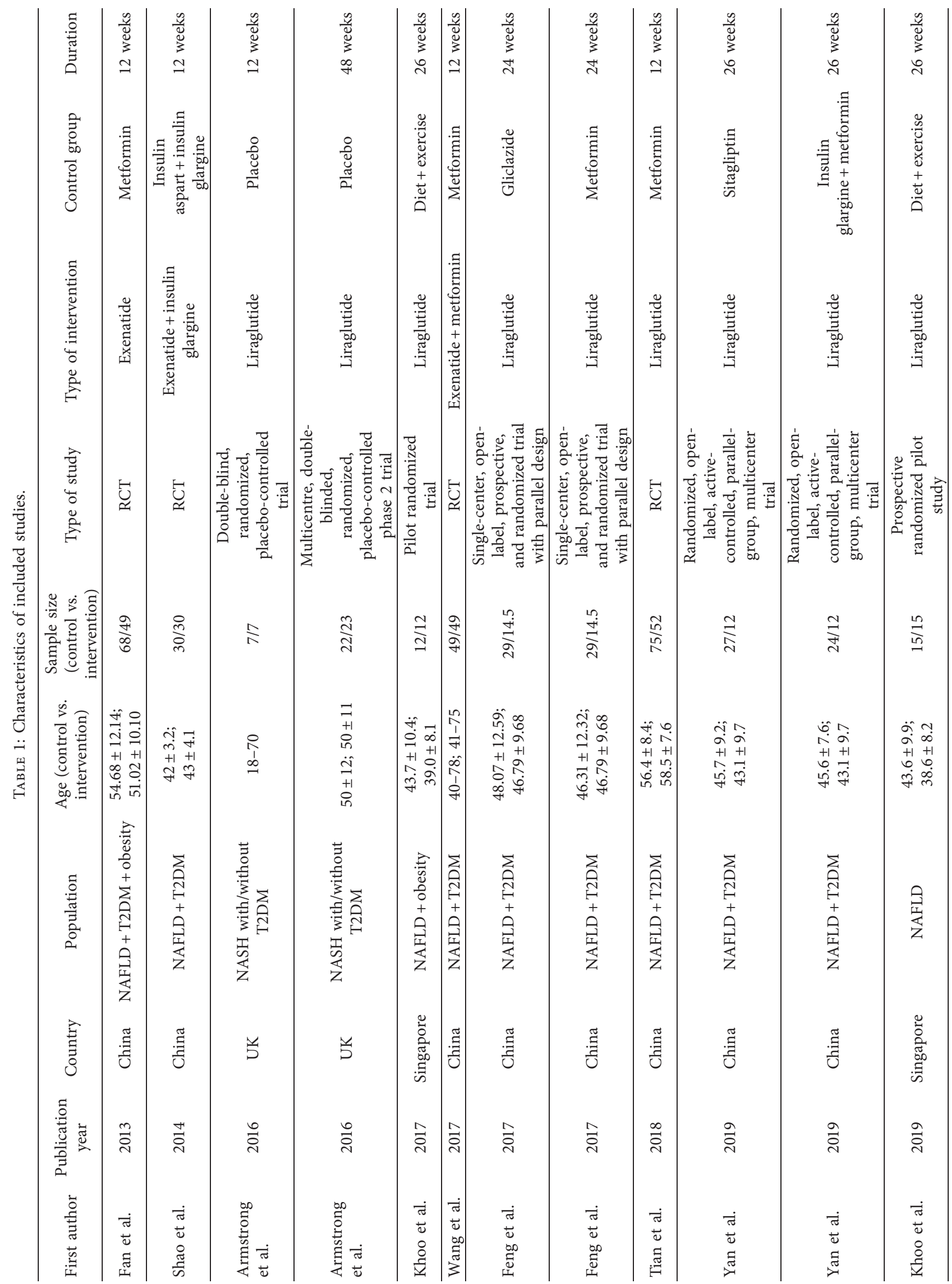




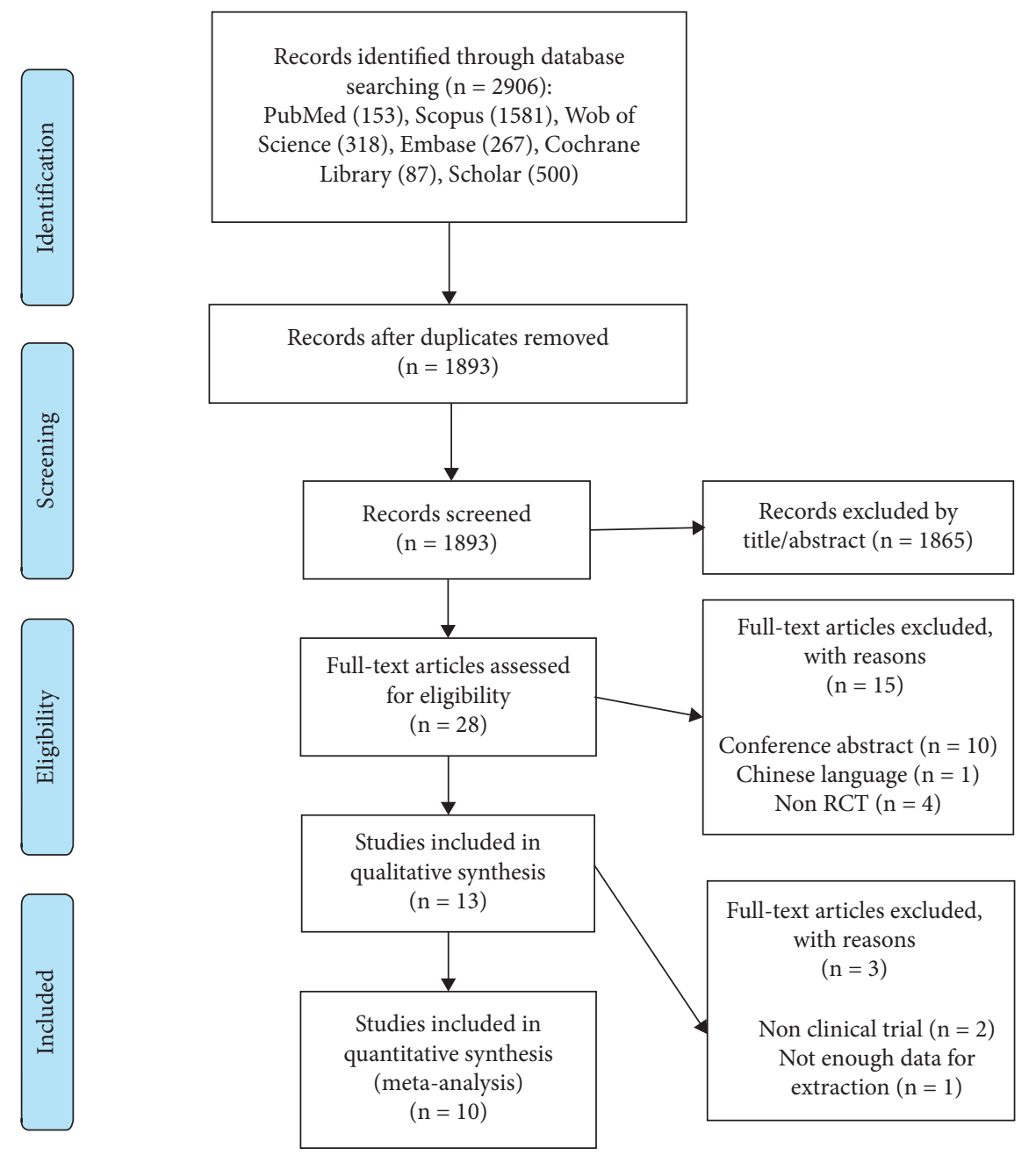

FIgURE 1: Flowchart of study selection method.

investigated by checking Cochrane's Q test $\left(I^{2}>50 \%\right.$, $P<0.1$ ) [24]. In cases with a high amount of statistical heterogeneity, a random-effects meta-regression was applied to find its potential source by confounders such as age, intervention duration, baseline body weight, and body mass index (BMI). Subgroup calculation was conducted according to the age ( $\geq 50$ years, $<50$ years), study duration ( $\leq 12$ weeks vs $>12$ weeks), BMI $(>30,<30)$, body weight ( $>85 \mathrm{~kg},<85 \mathrm{~kg}$ ), and type of intervention (GLP-1 vs. GLP1 plus other treatment) to detect the source of heterogeneity. Overall sensitivity analysis was performed to assess the dependence of pooled results by discarding each study in turn.

Estimation of the value of a correlation coefficient $(r)$ in each outcome was imputed from studies that reported the $\mathrm{SD}$ of change for each intervention group in the current meta-analysis. The following formula was used to determine the SD of change calculation among studies that did not provide sufficient information [24]:

$$
R=\left[\frac{R=\left(\mathrm{SD}_{\text {pre }}^{2}+\mathrm{SD}_{\text {post }}^{2}-\mathrm{SD}_{\text {Change }}^{2}\right)}{2 \times \mathrm{SD}_{\text {pre }} \times \mathrm{SD}_{\text {post }}}\right] \text {, }
$$

where $R$ was for TC: 0.81 , TG: 0.45 , HDL-c: 0.50 , LDL-C: 0.68, AST: 0.64, Alt: 0.62, GGT: 0.60, and ALP: 0.50. We also conducted a sensitivity analysis for outcomes (TG, AST, and ALT) with different values of $r$; TG (0.26 and 0.63), AST (0.20 and 0.77 ), and ALT (0.40 and 0.82$)$ to evaluate if the pooled results are sensitive to these levels.

2.6. The Grade Profile. The overall evaluation of the evidence relating to the outcomes was conducted by the Grading of Recommendations Assessment, Development, and Evaluation (GRADE) approach (Table 2) [25].

\section{Results}

3.1. Search Results and Study Selection. The flowchart explaining the method of selection and references obtained in the databases is shown in Figure 1. In total, 2906 articles were identified in the first phase of the literature search. After removal of duplicate studies $(n=1013)$, irrelevant studies according to the title and abstracts $(n=1865)$, different type of intervention $(n=4)$, conference abstracts $(n=10)$, and Chinese language $(n=1)$, thirteen potentially 
TABLE 2: Summary of findings.

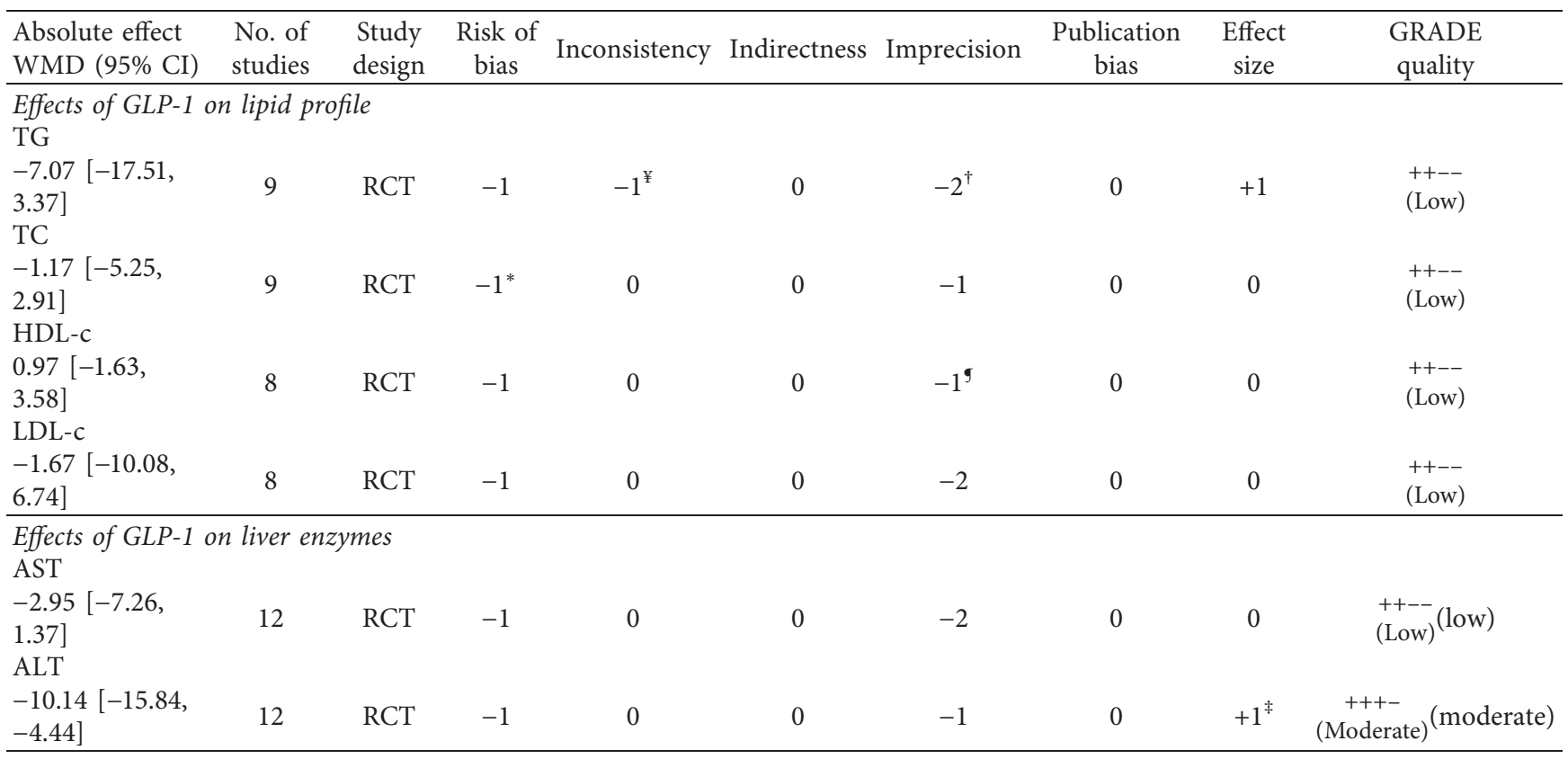

The symbols ++-- show the quality of evidence. Abbreviations: WMD, weighted mean difference; CI, confidence interval; RCT, randomized controlled trial; TG, triglycerides; TC, total cholesterol; HDL-c, high-density lipoprotein-cholesterol; LDL-c, low-density lipoprotein-cholesterol, AST, aspartate aminotransfrase; ALT, alanine aminotransfrase. * Downgraded one level as the moderate risk of bias. 'Downgraded one level as the confidence interval was moderate. ${ }^{\dagger}$ Downgraded two levels as the number of studies was $<5$ and imprecision was considerable. ${ }^{*}$ Upgraded one level due to considerable effect size. ${ }^{q}$ Downgraded one level as the statistical heterogeneity was $>50 \%$.

eligible studies were considered for full-text review. Subsequently, three articles were excluded for the following reasons: type of study and insufficient data reporting outcomes. Ultimately, ten studies were entered in the current meta-analysis.

3.2. Data Charachteristics. The main characteristics of the included trials are shown in Table 1. All of the RCTs were published between 2013 and 2019, were conducted in China [26-31], Singapore [32, 33], and UK $[34,35]$, and lasted 12 to 48 weeks. A total of 677 participants were aged between 18 to 70 years. Seven studies used Liraglutide as an intervention $[28,30-35]$, and two others $[26,27,29]$ used exenatide plus other treatments. The details of the quality assessment are shown in Figure 2.

3.3. The Effects of GLP-1 Receptor Agonists on Lipid Profile. The results of the meta-analysis regarding the influence of GLP-1 receptor agonists are shown in Figure 3. Pooled effect sizes indicated that receiving GLP-1 receptor agonists did not cause a statistically significant change in serum TG $(\mathrm{WMD}=-7.07,95 \% \mathrm{CI}=[-17.51,3.37], \quad P=0.18), \quad \mathrm{TC}$ $(\mathrm{WMD}=-1.17,95 \% \mathrm{CI}=[-5.25,2.91], P=0.57)$, HDL-C $(\mathrm{WMD}=0.97,95 \% \mathrm{CI}=[-1.63,3.58], P=0.46)$, and LDL-C $(\mathrm{WMD}=-1.67,95 \% \mathrm{CI}=[-10.08,6.74], \quad P=0.69) \quad$ in comparison with controls.
In addition, based on Cochrane's Q test, low degree of between-study heterogeneity was observed in TG $\left(I^{2}=0.0 \%\right.$, $P=0.6)$, TC $\left(I^{2}=27.2 \%, P=0.2\right)$, and HDL-C $\left(I^{2}=45.9 \%\right.$, $P<0.1)$. Conversely, LDL-C $\left(I^{2}=68.2 \%, P<0.1\right)$ had a high amount of statistical heterogeneity.

3.4. The Effects of GLP-1 Receptor Agonists on Liver Enzymes. Figure 4 presents the results of meta-analysis for liver enzymes. Treatment with GLP-1 receptor agonists lead to the amelioration of ALT serum concentration (WMD $=-10.14$, $95 \% \mathrm{CI}=[-15.84,-4.44], P<0.001), \mathrm{GGT}(\mathrm{WMD}=-11.53$, 95\% $\mathrm{CI}=[-15.21, \quad-7.85], \quad P<0.001), \quad$ and $\mathrm{ALP}$ $(\mathrm{WMD}=-8.29, \quad 95 \% \quad \mathrm{CI}=[-11.34, \quad-5.24], \quad P<0.001)$. However, serum AST level $(\mathrm{WMD}=-2.95,95 \% \mathrm{CI}=[-7.26$, 1.37], $P=0.18$ ) was not significantly affected following intervention.

Regarding between-study heterogeneity, Cochrane's Q test showed the following results: ALT $\left(I^{2}=80.6 \%, P<0.1\right)$, AST $\left(I^{2}=88.2 \%, P<0.1\right)$, ALP $\left(I^{2}=39.2 \%, P=0.19\right)$ and GGT $\left(I^{2}=53.6 \%, P<0.1\right)$.

3.5. Subgroup Analysis. As shown in Table 3, lipid profiles were not changed based on subgroup analysis. Conversely, AST and ALT were significantly affected when we conducted a subanalysis on duration ( $\leq 12$ weeks). However, serum ALT was significantly changed when subjects received GLP-1 agonists alone, and serum AST was reduced when they 


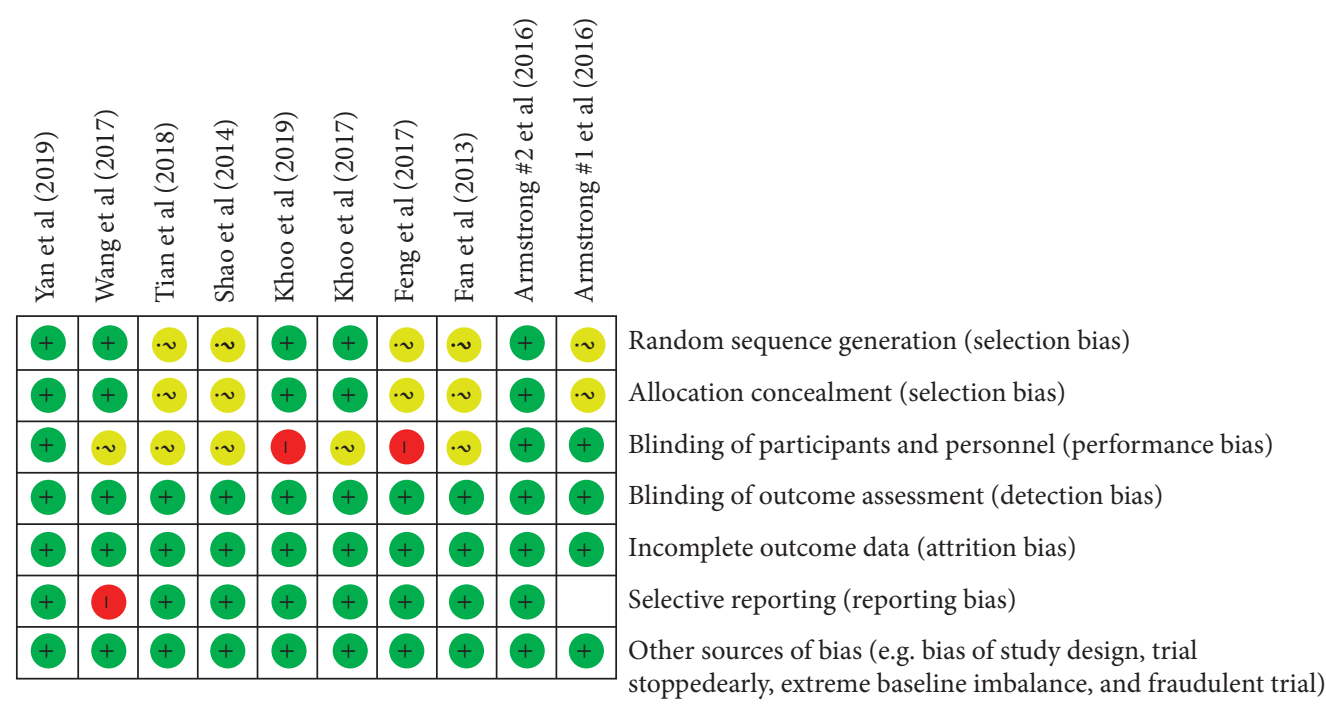

FIGURE 2: Details of quality assessment of the included papers.

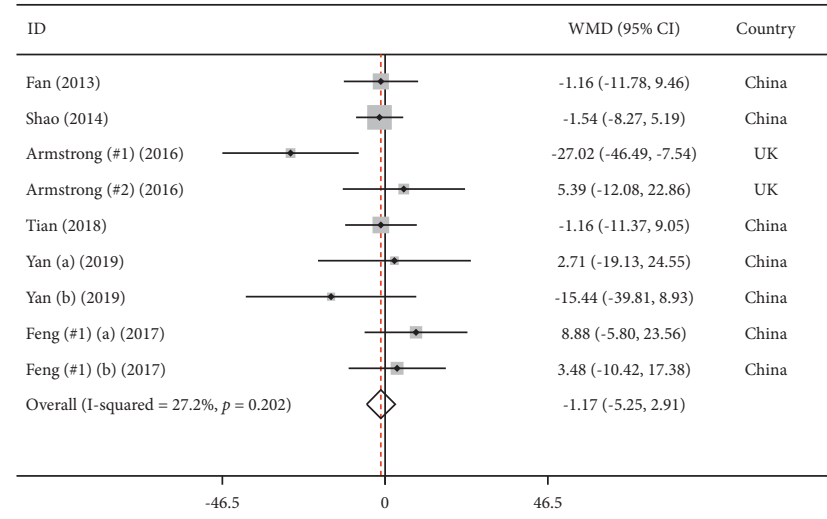

(a)

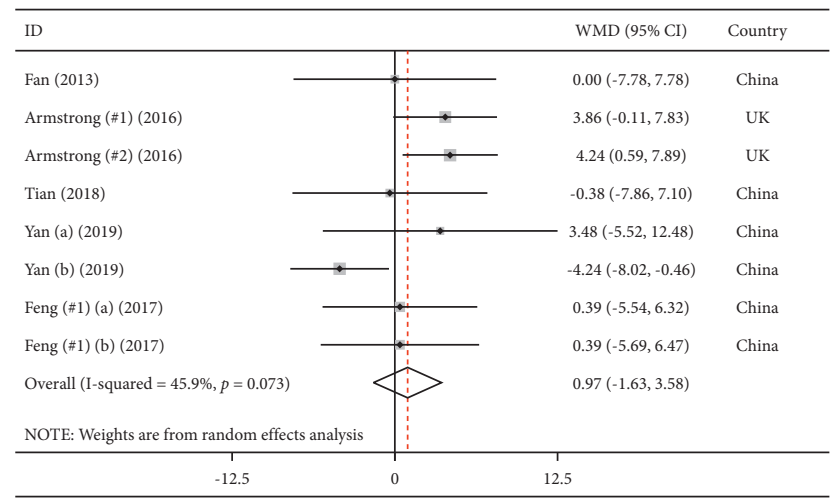

(c)

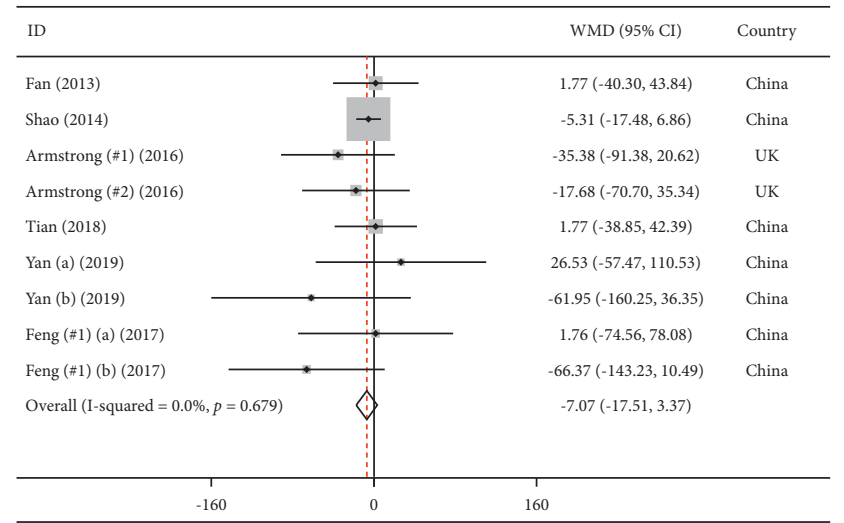

(b)

\begin{tabular}{|c|c|c|}
\hline ID & WMD $(95 \% \mathrm{CI})$ & Country \\
\hline Fan (2013) & $-1.16(-10.58,8.26)$ & China \\
\hline Armstrong (\#1) (2016) & $-28.95(-43.01,-14.89)$ & UK \\
\hline Armstrong (\#2) (2016) & $0.00(-18.24,18.24)$ & UK \\
\hline Tian (2018) & $-1.16(-10.23,7.91)$ & China \\
\hline Yan (a) (2019) & $3.48(-13.12,20.08)$ & China \\
\hline Yan (b) (2019) & $-8.10(-25.09,8.89)$ & China \\
\hline Feng (\#1) (a) (2017) & $9.66(-5.28,24.60)$ & China \\
\hline Feng (\#1) (b) (2017) & $12.74(-0.74,26.22)$ & China \\
\hline Overall $(\mathrm{I}-\mathrm{squared}=68.2 \%, p=0.003)$ & $-1.67(-10.08,6.74)$ & \\
\hline NOTE: Weights are from random effects analysis & & \\
\hline-43 & 43 & \\
\hline
\end{tabular}

(d)

FIGURE 3: Meta-analysis of weighted mean differences estimates for lipid profiles including (a) total cholesterol, (b) triglycerides, (c) HDLcholesterol, and (d) LDL-cholesterol in intervention and placebo groups (CI $=95 \%)$.

received another treatment along with GLP-1 agonists. In addition, the AST level was altered in older participants $(\geq 50$ years).
3.6. Sensitivity Analysis and Publication Bias. The sensitivity analysis was applied using "one-study-removed" strategy to investigate the influence of each study on the effect size. The 


\begin{tabular}{|c|c|c|c|}
\hline \multicolumn{2}{|l|}{ ID } & \multirow{2}{*}{$\begin{array}{c}\text { WMD }(95 \% \mathrm{CI}) \\
-2.78(-5.54,-0.02)\end{array}$} & \multirow{2}{*}{$\begin{array}{c}\text { Country } \\
\text { China }\end{array}$} \\
\hline Fan (2013) & + & & \\
\hline Shao (2014) & $\rightarrow$ & $-13.94(-20.19,-7.69)$ & China \\
\hline Armstrong (\#1) (2016) & - & $-36.00(-61.09,-10.91)$ & UK \\
\hline Armstrong (\#2) (2016) & & $-7.20(-22.01,7.61)$ & UK \\
\hline Khoo (2017) & 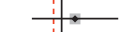 & $5.00(-11.01,21.01)$ & Singapore \\
\hline Wang (2017) & $\bullet$ & $-10.40(-11.82,-8.98)$ & China \\
\hline Tian (2018) & + & $-0.91(-3.34,1.52)$ & China \\
\hline Yan (a) (2019) & $\rightarrow$ & $6.90(-2.05,15.85)$ & China \\
\hline Yan (b) (2019) & : & $1.10(-9.64,11.84)$ & China \\
\hline Khoo (2019) & $\rightarrow$ & $8.00(-5.90,21.90)$ & Singapore \\
\hline Feng (\#1) (a) (2017) & 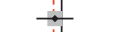 & $-2.47(-9.32,4.38)$ & China \\
\hline Feng (\#1) (b) (2017) & 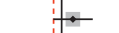 & $4.25(-3.07,11.57)$ & China \\
\hline Overall (I-squared $=88.2 \%, p=0.000)$ & 10 & $-2.95(-7.26,1.37)$ & \\
\hline \multicolumn{4}{|c|}{ NOTE: Weights are from random effects analysis } \\
\hline-61.1 & 0 & 61.1 & \\
\hline
\end{tabular}

(a)

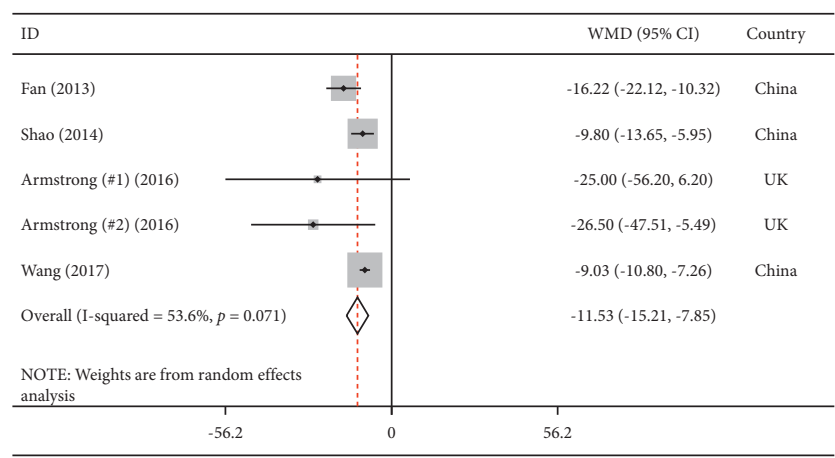

(c)

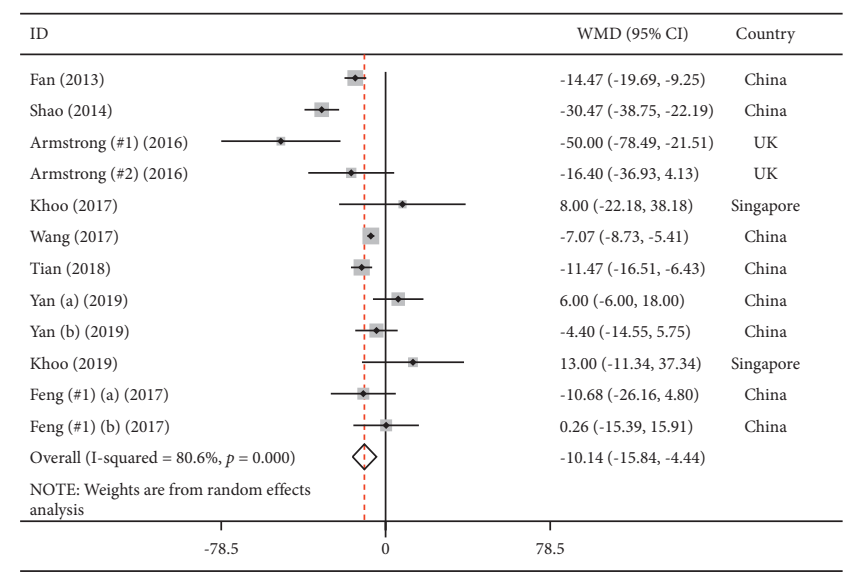

(b)

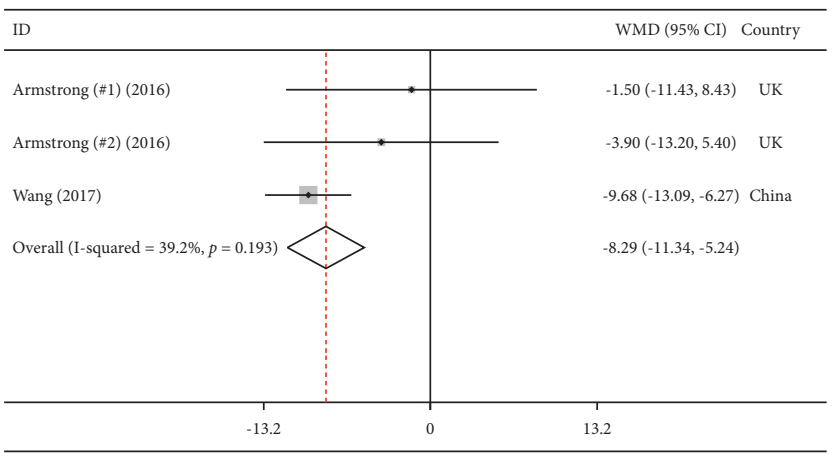

(d)

Figure 4: Meta-analysis of weighted mean differences estimates for liver enzymes including (a) aspartate aminotransferase, (b) alanine aminotransferase, (c) gamma-glutamyltransferase, and (d) alkaline phosphatase in intervention and placebo groups $(\mathrm{CI}=95 \%)$.

results of sensitivity analysis displayed that the pooled results of interested outcomes were not sensitive to each study. Additionaly, we checked triglycerides, aspartate aminotransferase, alanine aminotransferase (TG, AST, and ALT) with different values of $r$. The significance of the results of TG $\quad\left(r=0.26: \quad I^{2}=0.0 \%, \quad P=0.22 ; \quad r=0.63: \quad I^{2}=0.0 \%\right.$, $P=0.13), \quad$ AST $\quad\left(r=0.20: I^{2}=83.7 \%, \quad P=0.18 ; \quad r=0.77\right.$ : $\left.I^{2}=99.4 \%, \quad P=0.05\right), \quad$ and $\operatorname{ALT} \quad\left(r=0.40: \quad I^{2}=76.3 \%\right.$, $\left.P<0.001 ; r=0.82: I^{2}=86.5 \%, P=0.001\right)$ was independent of different values of $r$. Due to the minimum number of studies required for the assessment of publication bias by funnel plot being 10 and for Egger's test being 20, these tools for detection of publication bias would not be meaningful with so few studies and therefore were not performed.

\section{Discussion}

This meta-analysis showed that the combined available studies, including liraglutide and exenatide, showed an improvement in the liver enzymes of patients with NAFLD but that the lipid profile was unchanged. This suggests that GLP-1 agonists may have utility in the treatment of NAFLD or at least prevention of further progression. Similarly, hepatic histological features in patients with nonalcoholic steatohepatitis (NAFLD with additional inflammation) were improved in the liraglutide group compared to placebo (hepatocyte ballooning (61\% vs. $32 \%$ ) and steatosis (83\% vs. 45\%) [34]. Moreover, in a recent meta-analysis of four clinical trials, histological improvement was demonstrated [36]. The mechanism by which liraglutide may improve NAFLD could be through inhibiting the NLRP3 inflammasome and pyroptosis activation through mitophagy [37]. Currently, there is no recognized therapeutic agent for the treatment of NAFLD [38]; however, whilst the studies with these GLP-1 agonists may be encouraging, they are of too short a study duration to know if their effects are maintained or that they have continued clinical therapeutic utility.

Liraglutide is reported to have a cholesterol-lowering effect though the mechanism is unclear [39], and others have shown an improvement in lipids in nondiabetic subjects $[20,40]$. In this meta-analysis, there was no effect of GLP-1 agonists on any of the lipid parameters, including TG, TC, HDL-C (where the heterogeneity between studies was low), and LDL-C (where the heterogeneity between studies was high). This suggests that GLP-1 agonists do not have a direct effect on lipid metabolism in NAFLD and that the lipid changes reported in the literature may have been indirectly due to associated weight loss through the satiety effects of the GLP-1 agonists such as liraglutide [17].

The strength of this study was that it focused on randomized clinical trials that would increase its power. This meta-analysis has a number of limitations. Firstly, the effects 
TABLe 3: The results of subgroup analysis for serum TC, TG, HDL-C, LDL-C, AST, and ALT.

\begin{tabular}{|c|c|c|c|c|c|c|c|}
\hline & Subgroup & Study & $\begin{array}{l}\text { WMD } \\
(95 \% \text { CI })\end{array}$ & $\begin{array}{c}P \\
\text { value }\end{array}$ & $\begin{array}{l}\text { Heterogeneity } \\
\left(I^{2}\right)\end{array}$ & $\begin{array}{l}\text { Meta- } \\
\text { regression }\end{array}$ & $\begin{array}{c}\text { Test of group } \\
\text { differences } P>Q_{-} b\end{array}$ \\
\hline \multicolumn{8}{|l|}{$T G$} \\
\hline \multirow[t]{2}{*}{ Age } & $\geq 50$ years old & 3 & $\begin{array}{c}-2.76 \\
(-28.35,22.83)\end{array}$ & 0.83 & 0.0 & \multirow[t]{2}{*}{-} & \multirow[t]{2}{*}{0.718} \\
\hline & $<50$ years old & 6 & $-7.93(-19.36,3.5)$ & 0.17 & 3.7 & & \\
\hline \multirow[b]{2}{*}{ Duration } & $\leq 12$ weeks & 4 & $\begin{array}{c}-5.47 \\
(-16.48,5.55)\end{array}$ & 0.33 & 0.0 & \multirow[b]{2}{*}{-} & \multirow[b]{2}{*}{0.373} \\
\hline & $>12$ weeks & 5 & $\begin{array}{c}-21.10 \\
(-17.51,3.37)\end{array}$ & 0.20 & 0.0 & & \\
\hline \multirow{2}{*}{ Baseline BMI } & $>30$ & 4 & $\begin{array}{c}-7.31 \\
(-18.72,4.10)\end{array}$ & 0.21 & 0.0 & \multirow[b]{2}{*}{-} & \multirow{2}{*}{0.920} \\
\hline & $<30$ & 4 & $\begin{array}{c}-5.86 \\
(-31.57,19.85)\end{array}$ & 0.65 & 0.0 & & \\
\hline \multirow{2}{*}{$\begin{array}{l}\text { Baseline body } \\
\text { weight }\end{array}$} & $>85 \mathrm{~kg}$ & 4 & $\begin{array}{c}-7.31 \\
(-18.72,4.10)\end{array}$ & 0.21 & 0.0 & \multirow{2}{*}{-} & \multirow{2}{*}{0.920} \\
\hline & $<85 \mathrm{~kg}$ & 4 & $\begin{array}{c}-5.86 \\
(-31.57,19.85)\end{array}$ & 0.65 & 0.0 & & \\
\hline \multirow{2}{*}{$\begin{array}{l}\text { Intervention } \\
\text { type }\end{array}$} & GLP-1 agonists & 8 & $\begin{array}{c}-11.96 \\
(-32.24,8.32)\end{array}$ & 0.24 & 0.0 & \multirow{2}{*}{-} & \multirow{2}{*}{0.582} \\
\hline & $\begin{array}{c}\text { GLP-1 agonists + other } \\
\text { treatment }\end{array}$ & 1 & $\begin{array}{c}-5.31 \\
(-17.48,6.86) \\
\end{array}$ & 0.39 & - & & \\
\hline \multicolumn{8}{|l|}{$T C$} \\
\hline \multirow{2}{*}{ Age } & $\geq 50$-years old & 3 & $-0.17(-6.95,6.61)$ & 0.96 & 0.0 & \multirow{2}{*}{-} & \multirow{2}{*}{0.719} \\
\hline & $<50$ years old & 6 & $-1.73(-6.84,3.37)$ & 0.50 & 51.9 & & \\
\hline \multirow{2}{*}{ Duration } & $\leq 12$ weeks & 4 & $-2.93(-7.74,1.88)$ & 0.23 & 52.1 & \multirow{2}{*}{-} & \multirow{2}{*}{0.175} \\
\hline & $>12$ weeks & 5 & $3.35(-4.34,11.05)$ & 0.39 & 0.0 & & \\
\hline Baseline $\mathrm{BMI}$ & $>30$ & 4 & $-3.39(-9.00,2.21)$ & 0.23 & 51.0 & 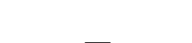 & \\
\hline Baselıne BMI & $<30$ & 4 & $1.33(-4.61,7.28)$ & 0.65 & 0.0 & - & 0.256 \\
\hline Baseline body & $>85 \mathrm{~kg}$ & 4 & $-3.39(-9.00,2.21)$ & 0.23 & 51.0 & & \\
\hline weight & $<85 \mathrm{~kg}$ & 4 & $1.33(-4.61,7.28)$ & 0.65 & 0.0 & - & 0.256 \\
\hline & GLP-1 agonists & 8 & $-0.95(-6.08,4.18)$ & 0.71 & 36.2 & & \\
\hline $\begin{array}{l}\text { Intervention } \\
\text { type }\end{array}$ & $\begin{array}{l}\text { GLP-1 agonists + other } \\
\text { treatment }\end{array}$ & 1 & $-1.54(-8.27,5.19)$ & - & - & - & 0.891 \\
\hline$H D L-C$ & & & & & & & \\
\hline Age & $\geq 50$ years old & 3 & $2.84(-0.18,5.86)$ & 0.06 & 0.0 & - & 0307 \\
\hline Age & $<50$ years old & 5 & $0.40(-3.18,3.98)$ & 0.82 & 55.8 & - & 0.307 \\
\hline & $\leq 12$ weeks & 3 & $2.43(-0.76,5.63)$ & 0.13 & 0.0 & & \\
\hline Duration & $>12$ weeks & 5 & $0.56(-3.21,4.35)$ & 0.76 & 61.8 & - & 0.460 \\
\hline Baseline BMI & $>30$ & 4 & $1.61(-3.03,6.26)$ & 0.49 & 76.1 & - & 0619 \\
\hline & $<30$ & 4 & $0.16(-3.17,3.50)$ & 0.92 & 0.0 & - & 0.019 \\
\hline Baseline body & $>85 \mathrm{~kg}$ & 4 & $1.61(-3.03,6.26)$ & 0.49 & 76.1 & & 0610 \\
\hline weight & $<85 \mathrm{~kg}$ & 4 & $0.16(-3.17,3.50)$ & 0.92 & 0.0 & - & 0.619 \\
\hline$L D L-C$ & & & & & & & \\
\hline & $\geq 50$ years old & 3 & $-1.02(-7.17,5.12)$ & 0.74 & 0.0 & & \\
\hline Age & $<50$ years old & 5 & $\begin{array}{c}-2.22 \\
(-18.06,13.60)\end{array}$ & 0.78 & 81.8 & $2.78)$ & 0.890 \\
\hline Duration & $\leq 12$ weeks & 3 & $\begin{array}{c}-9.48 \\
(-24.70,5.74)\end{array}$ & 0.22 & 83.8 & $0.36(-0.37$ & 0.097 \\
\hline & $>12$ weeks & 5 & $4.79(-2.48,12.07)$ & 0.19 & 5.8 & $1.40)$ & \\
\hline Baseline BMI & $>30$ & 4 & $\begin{array}{c}-8.96 \\
(-24.42,6.50)\end{array}$ & 0.25 & 71.9 & $-3.43(-7.10$ & 0.155 \\
\hline & $<30$ & 4 & $3.26(-3.46,10.00)$ & 0.34 & 30.3 & & \\
\hline Baseline body & $>85 \mathrm{~kg}$ & 4 & $\begin{array}{c}-8.96 \\
(-24.42,6.50)\end{array}$ & 0.25 & 71.9 & $-0.59(-1.36$ & \\
\hline weight & $<85 \mathrm{~kg}$ & 4 & $3.26(-3.46,10.00)$ & 0.34 & 30.3 & $0.18)$ & \\
\hline$A S T$ & & & & & & & \\
\hline Age & $\geq 50$ years old & 4 & $\begin{array}{c}-5.04 \\
(-11.24,1.15)\end{array}$ & 0.11 & 95.2 & $-0.31(-1.22$ & 0.663 \\
\hline & $<50$ years old & 8 & $-1.60(-8.97,5.77)$ & 0.67 & 80.4 & $0.58)$ & \\
\hline
\end{tabular}


TABle 3: Continued.

\begin{tabular}{|c|c|c|c|c|c|c|c|}
\hline & Subgroup & Study & $\begin{array}{c}\text { WMD } \\
(95 \% \mathrm{CI}) \\
\end{array}$ & $\begin{array}{c}P \\
\text { value }\end{array}$ & $\begin{array}{l}\text { Heterogeneity } \\
\left(I^{2}\right)\end{array}$ & $\begin{array}{c}\text { Meta- } \\
\text { regression }\end{array}$ & $\begin{array}{c}\text { Test of group } \\
\text { differences } P>Q_{-} b\end{array}$ \\
\hline \multirow{2}{*}{ Duration } & $\leq 12$ weeks & 5 & $\begin{array}{c}-8.07(-13.86 \\
-2.29)\end{array}$ & 0.006 & 94.4 & \multirow{2}{*}{$\begin{array}{c}0.36(-0.22 \\
0.95)\end{array}$} & \multirow[t]{2}{*}{0.031} \\
\hline & $>12$ weeks & 7 & $1.84(-1.59,5.28)$ & 0.29 & 0.2 & & \\
\hline \multirow[b]{2}{*}{$\begin{array}{l}\text { Intervention } \\
\text { type }\end{array}$} & GLP-1 agonists & 10 & $-0.35(-3.44,2.75)$ & 0.82 & 47.6 & \multirow[b]{2}{*}{-} & \multirow[b]{2}{*}{0.000} \\
\hline & $\begin{array}{l}\text { GLP-1 agonists + other } \\
\text { treatment }\end{array}$ & 2 & $\begin{array}{c}-10.81(-13.03 \\
-8.59)\end{array}$ & $<0.001$ & 14.7 & & \\
\hline \multicolumn{8}{|l|}{$A L T$} \\
\hline \multirow{2}{*}{ Age } & $\geq 50$ years old & 4 & $\begin{array}{c}-10.71 \\
(-15.32,-6.11)\end{array}$ & $<0.001$ & 71.1 & \multirow{2}{*}{$\begin{array}{c}-0.30(-1.86 \\
1.26)\end{array}$} & \multirow{2}{*}{0.997} \\
\hline & $<50$ years old & 8 & $\begin{array}{c}-8.47 \\
(-22.18,5.24)\end{array}$ & 0.22 & 88.1 & & \\
\hline \multirow[t]{2}{*}{ Duration } & $\leq 12$ weeks & 5 & $\begin{array}{c}-18.12 \\
(-27.34,-8.91)\end{array}$ & $<0.001$ & 94.2 & \multirow{2}{*}{$\begin{array}{c}0.53(-0.41 \\
1.48)\end{array}$} & \multirow[t]{2}{*}{0.003} \\
\hline & $>12$ weeks & 7 & $-2.05(-8.43,4.31)$ & 0.52 & 20.7 & & \\
\hline \multirow{2}{*}{$\begin{array}{l}\text { Intervention } \\
\text { type }\end{array}$} & GLP-1 agonists & 10 & $\begin{array}{c}-7.69 \\
(-14.20,-1.18)\end{array}$ & 0.02 & 64.7 & \multirow{2}{*}{ - } & \multirow{2}{*}{0.378} \\
\hline & $\begin{array}{l}\text { GLP-1 agonists + other } \\
\text { treatment }\end{array}$ & 2 & $\begin{array}{c}-18.40 \\
(-41.32,4.52)\end{array}$ & 0.11 & 96.6 & & \\
\hline
\end{tabular}

of GLP-1 therapy on liver enzymes and the lipid profile in NAFLD were not the primary aim of the clinical trials and the studies were not powered for this. Secondly, there were only 12 trials with relatively few subjects available to be analyzed, giving a modest though robust number of subjects to undertake the analysis. The meta-analysis was also limited in that only two studies were with exenatide and the remainder was with liraglutide and no studies were available for the newer GLP-1 agonists such as semaglutide. Since GLP-1 agonists have differing structures and potencies, their effects on liver enzymes are also likely to be different [41].

\section{Conclusion}

The results of this meta-analysis suggest that GLP-1 agonist treatment significantly reduces the liver enzymes ALT, GGT, and ALP, though AST was no different in patients with NAFLD; however, the lipid profile is unaffected.

\section{Appendix}

\section{Search String Employed for the Systematic Review}

(INDEXTERMS ("GLP-1 analog" OR "glucagon-like peptide-1 analog" OR "GLP-1 receptor agonist" OR "glucagon-like peptide-1 receptor agonist" OR "glp-1 receptor agonists" OR "glp1 receptor agonist” OR "glucagon-like" OR exenatide OR lixisenatide OR eperzan OR tanzeum OR albiglutide OR dulaglutide OR liraglutide OR semaglutide OR taspoglutide OR tanzeum OR trulicity OR byetta OR bydureon OR victoza OR adlyxin OR ozemoic OR saxenda OR bydureon OR "ITCA 650" OR "Exendin4" OR "Exendin 4" OR byetta OR adlyxin OR lyxumia OR "rGLP-1 protein" OR ozempic) OR TITLE-ABS-KEY ("GLP-1 analog” OR "glucagon-like peptide-1 analog” OR "GLP-1 receptor agonist" OR "glucagon-like peptide-1 receptor agonist” OR “glp-1 receptor agonists” OR “glp1 receptor agonist” OR "glucagon-like” OR exenatide OR lixisenatide OR eperzan OR tanzeum OR albiglutide OR dulaglutide OR liraglutide OR semaglutide OR taspoglutide OR tanzeum OR trulicity OR byetta OR bydureon OR victoza $O R$ adlyxin OR ozemoic OR saxenda OR bydureon OR “ITCA 650" OR “Exendin-4" OR “Exendin 4" OR byetta OR adlyxin OR lyxumia OR "rGLP-1 protein” OR ozempic)) AND (INDEXTERMS (NASH OR Liver OR "Fatty Liver" OR steatohepatitis OR "Steatosis of Liver" OR "Visceral Steatosis" OR steatosis OR "Liver Steatosis" OR "Non-alcoholic Fatty Liver Disease" OR "Non alcoholic Fatty Liver Disease" OR "NAFLD" OR "Nonalcoholic Fatty Liver Disease" OR nonalcoholic OR "Non alcoholic" OR "Nonalcoholic Fatty" OR "Non-alcoholic Fatty" OR "Nonalcoholic Fatty Liver" OR "Nonalcoholic Fatty Livers" OR “Nonalcoholic Steatohepatitis" OR steatohepatitis OR steatotic) OR TITLE-ABS-KEY (NASH OR Liver OR "Fatty Liver" OR steatohepatitis OR "Steatosis of Liver" OR "Visceral Steatosis" OR steatosis OR "Liver Steatosis" OR "Non-alcoholic Fatty Liver Disease" OR "Non alcoholic Fatty Liver Disease" OR "NAFLD" OR "Nonalcoholic Fatty Liver Disease" OR nonalcoholic OR "Non alcoholic" OR "Nonalcoholic Fatty" OR "Non-alcoholic Fatty" OR "Nonalcoholic Fatty Liver" OR "Nonalcoholic Fatty Livers" OR "Nonalcoholic Steatohepatitis" OR steatohepatitis OR steatotic)) AND (INDEXTERMS ("randomized clinical trial" OR "Randomized controlled trial" OR "random allocation" OR randomized OR "clinical trial" OR random* OR trial OR blind OR "controlled trial" OR "controlled study" OR "randomized trial” OR "clinical study") OR TITLE-ABS-KEY ("randomized clinical trial" OR "Randomized controlled trial" OR "random allocation" OR randomized OR "clinical trial" OR random* OR trial OR blind OR "controlled trial” OR "controlled study" OR "randomized trial" OR "clinical study")). 


\section{Data Availability}

There are no raw data associated with this review article.

\section{Conflicts of Interest}

The authors declare that there are no conflicts of interest.

\section{References}

[1] A. R. Araújo, N. Rosso, G. Bedogni, C. Tiribelli, and S. Bellentani, "Global epidemiology of nonalcoholic fatty liver disease/nonalcoholic steatohepatitis: what we need in the future," Liver International, vol. 38, pp. 47-51, 2018.

[2] H. Hagström, P. Nasr, M. Ekstedt et al., "Risk for development of severe liver disease in lean patients with nonalcoholic fatty liver disease: a long-term follow-up study," Hepatology communications, vol. 2, no. 1, pp. 48-57, 2018.

[3] J. Wise, "Pioglitazone seems safe and effective for patients with fatty liver disease and diabetes," BMJ, vol. 353, p. i3435, 2016.

[4] V. W.-S. Wong, W.-K. Chan, S. Chitturi et al., "Asia-pacific working party on non-alcoholic fatty liver disease guidelines 2017-part 1: definition, risk factors and assessment," Journal of Gastroenterology and Hepatology, vol. 33, no. 1, pp. 70-85, 2018.

[5] K. J. P. Schwenger, Nonalcoholic Fatty Liver Disease: Investigating the Impact of Bariatric Care and the Role of Immune Function, University of Toronto, Toronto, Canada, 2018.

[6] Z. M. Younossi, R. Loomba, M. E. Rinella et al., "Current and future therapeutic regimens for nonalcoholic fatty liver disease and nonalcoholic steatohepatitis," Hepatology, vol. 68, no. 1, pp. 361-371, 2018.

[7] H. Yaribeygi, T. Sathyapalan, and A. Sahebkar, "Molecular mechanisms by which GLP-1 RA and DPP-4i induce insulin sensitivity," Life Sciences, vol. 234, Article ID 116776, 2019.

[8] H. Yaribeygi, M. Maleki, T. Sathyapalan, T. Jamialahmadi, and A. Sahebkar, "Anti-inflammatory potentials of incretinbased therapies used in the management of diabetes," Life Sciences, vol. 241, Article ID 117152, 2020.

[9] H. Yaribeygi, M. Ashrafizadeh, N. C. Henney, T. Sathyapalan, T. Jamialahmadi, and A. Sahebkar, "Neuromodulatory effects of anti-diabetes medications: a mechanistic review," Pharmacological Research, vol. 152, pp. 1096-1186, 2020.

[10] S. Radbakhsh, S. L. Atkin, L. E. Simental-Mendia, and A. Sahebkar, "The role of incretins and incretin-based drugs in autoimmune diseases," International Immunopharmacology, vol. 98, Article ID 107845, 2021.

[11] H. Yaribeygi, S. L. Atkin, F. Montecucco, T. Jamialahmadi, and A. Sahebkar, "Renoprotective effects of incretin-based therapy in diabetes mellitus," BioMed Research International, vol. 2021, Article ID 8163153, 7 pages, 2021.

[12] H. Yaribeygi, F. R. Farrokhi, M. A. Abdalla et al., "The effects of glucagon-like peptide-1 receptor agonists and dipeptydilpeptidase- 4 inhibitors on blood pressure and cardiovascular complications in diabetes," Journal of Diabetes Research, vol. 2021, Article ID 6518221, 10 pages, 2021.

[13] H. Yaribeygi, N. Katsiki, A. E. Butler, and A. Sahebkar, "Effects of antidiabetic drugs on NLRP3 inflammasome activity, with a focus on diabetic kidneys," Drug Discovery Today, vol. 24, no. 1, pp. 256-262, 2019.

[14] H. Yaribeygi, A. Rashidy-Pour, S. L. Atkin, T. Jamialahmadi, and A. Sahebkar, "GLP-1 mimetics and cognition," Life Sciences, vol. 264, p. 118645, 2021.
[15] H. Yaribeygi, M. Maleki, T. Sathyapalan, T. Jamialahmadi, and A. Sahebkar, "Antioxidative potentials of incretin-based medications: a review of molecular mechanisms," Oxidative Medicine and Cellular Longevity, vol. 2021, Article ID 9959320, 9 pages, 2021.

[16] G. Ranjbar, D. P. Mikhailidis, and A. Sahebkar, "Effects of newer antidiabetic drugs on nonalcoholic fatty liver and steatohepatitis: think out of the box!," Metabolism, vol. 101, p. 154001, 2019.

[17] T. Ohki, A. Isogawa, M. Iwamoto et al., "The effectiveness of liraglutide in nonalcoholic fatty liver disease patients with type 2 diabetes mellitus compared to sitagliptin and pioglitazone," The ScientificWorld Journal, vol. 2012, Article ID 496453, 8 pages, 2012.

[18] F. Angelico, M. Del Ben, R. Conti et al., "Insulin resistance, the metabolic syndrome, and nonalcoholic fatty liver disease," Journal of Clinical Endocrinology \& Metabolism, vol. 90, no. 3, pp. 1578-1582, 2005.

[19] W. Zhu, P.-P. Feng, K. He, S.-W. Li, and J.-P. Gong, "Liraglutide protects non-alcoholic fatty liver disease via inhibiting NLRP3 inflammasome activation in a mouse model induced by high-fat diet," Biochemical and Biophysical Research Communications, vol. 505, no. 2, pp. 523-529, 2018.

[20] H. Kahal, G. Abouda, A. S. Rigby, A. M. Coady, E. S. Kilpatrick, and S. L. Atkin, "Glucagon-like peptide-1 analogue, liraglutide, improves liver fibrosis markers in obese women with polycystic ovary syndrome and nonalcoholic fatty liver disease," Clinical Endocrinology, vol. 81, no. 4, pp. 523-528, 2014.

[21] D. Moher, A. Liberati, J. Tetzlaff, and D. G. J. A. Altman, "Preferred reporting items for systematic reviews and metaanalyses," The PRISMA Statement, vol. 151, no. 4, pp. 264-269, 2009.

[22] M. Borenstein, L. V. Hedges, J. P. Higgins, and H. R. Rothstein, Introduction to Meta-Analysis, John Wiley \& Sons, Hoboken, NJ, USA, 2011.

[23] J. P. T. Higgins, D. G. Altman, P. C. Gotzsche et al., "The Cochrane collaboration's tool for assessing risk of bias in randomised trials," BMJ, vol. 343, p. d5928, 2011.

[24] J. Higgins, J. Thomas, J. Chandler et al., Cochrane Handbook for Systematic Reviews of Interventions, Cochrane Handbook, London, UK, 2019.

[25] G. H. Guyatt, A. D. Oxman, G. E. Vist et al., "GRADE: an emerging consensus on rating quality of evidence and strength of recommendations," BMJ, vol. 336, no. 7650, pp. 924-926, 2008.

[26] H. Fan, Q. Pan, Y. Xu, and X. Yang, "Exenatide improves type 2 diabetes concomitant with non-alcoholic fatty liver disease," Arquivos Brasileiros de Endocrinologia \& Metabologia, vol. 57, no. 9, pp. 702-708, 2013.

[27] N. Shao, H. Y. Kuang, M. Hao, X. Y. Gao, W. J. Lin, and W. Zou, "Benefits of exenatide on obesity and non-alcoholic fatty liver disease with elevated liver enzymes in patients with type 2 diabetes," Diabetes, vol. 30, no. 6, pp. 521-529, 2014.

[28] W. Feng, C. Gao, Y. Bi et al., "Randomized trial comparing the effects of gliclazide, liraglutide, and metformin on diabetes with non-alcoholic fatty liver disease," Journal of Diabetes, vol. 9, no. 8, pp. 800-809, 2017.

[29] D. J. J. M. U. Wang, "Effect of exenatide combined with metformin therapy on insulin resistance, liver function and inflammatory response in patients with type 2 diabetes mellitus combined with NAFLD," Journal of Hainan Medical University, vol. 23, no. 18, pp. 44-47, 2017. 
[30] F. Tian, Z. Zheng, D. Zhang, S. He, and J. Shen, "Efficacy of liraglutide in treating type 2 diabetes mellitus complicated with non-alcoholic fatty liver disease," Bioscience Reports, vol. 38, 2018.

[31] J. Yan, B. Yao, H. Kuang et al., "Liraglutide, sitagliptin, and insulin glargine added to metformin: the effect on body weight and intrahepatic lipid in patients with type 2 diabetes mellitus and nonalcoholic fatty liver disease," Hepatology, vol. 69, no. 6, pp. 2414-2426, 2019.

[32] J. Khoo, J. Hsiang, R. Taneja, N. M. Law, and T. L. Ang, "Comparative effects of liraglutide $3 \mathrm{mg}$ vs structured lifestyle modification on body weight, liver fat and liver function in obese patients with non-alcoholic fatty liver disease: a pilot randomized trial," Diabetes, Obesity and Metabolism, vol. 19, no. 12, pp. 1814-1817, 2017.

[33] J. Khoo, M. Ho, S. Kam et al., "Comparing effects of liraglutide-induced weight loss versus lifestyle modification on liver fat content and plasma acylcarnitine levels in obese adults with nonalcoholic fatty liver disease," Obesity Facts, vol. 12, p. 61, 2019.

[34] M. J. Armstrong, P. Gaunt, G. P. Aithal et al., "Liraglutide safety and efficacy in patients with non-alcoholic steatohepatitis (LEAN): a multicentre, double-blind, randomised, placebo-controlled phase 2 study," The Lancet, vol. 387, no. 10019, pp. 679-690, 2016.

[35] M. J. Armstrong, D. Hull, K. Guo et al., "Glucagon-like peptide 1 decreases lipotoxicity in non-alcoholic steatohepatitis," Journal of Hepatology, vol. 64, no. 2, pp. 399-408, 2016.

[36] X. Lv, Y. Dong, L. Hu, F. Lu, C. Zhou, and S. Qin, “Glucagonlike peptide-1 receptor agonists (GLP-1 RAs) for the management of nonalcoholic fatty liver disease (NAFLD): a systematic review," Endocrinology, Diabetes \& Metabolism, vol. 3, no. 3, Article ID e00163, 2020.

[37] X. Yu, M. Hao, Y. Liu et al., "Liraglutide ameliorates nonalcoholic steatohepatitis by inhibiting NLRP3 inflammasome and pyroptosis activation via mitophagy," European Journal of Pharmacology, vol. 864, Article ID 172715, 2019.

[38] Y. Sumida, M. Yoneda, K. Tokushige et al., "Antidiabetic therapy in the treatment of nonalcoholic steatohepatitis," International Journal of Molecular Sciences, vol. 21, no. 6, 2020.

[39] K. Aoki, H. Kamiyama, M. Takihata et al., "Effect of liraglutide on lipids in patients with type 2 diabetes: a pilot study," Endocrine Journal, vol. 67, no. 9, pp. 957-962, 2020.

[40] H. Kahal, A. Aburima, T. Ungvari et al., "The effects of treatment with liraglutide on atherothrombotic risk in obese young women with polycystic ovary syndrome and controls," BMC Endocrine Disorders, vol. 15, no. 1, p. 14, 2015.

[41] M. S. Capehorn, A.-M. Catarig, J. K. Furberg et al., "Efficacy and safety of once-weekly semaglutide $1.0 \mathrm{mg}$ vs once-daily liraglutide $1.2 \mathrm{mg}$ as add-on to 1-3 oral antidiabetic drugs in subjects with type 2 diabetes (SUSTAIN 10)," Diabetes \& Metabolism, vol. 46, no. 2, pp. 100-109, 2020. 